\title{
Promoter specificity and interactions between early and late Arabidopsis heat shock factors
}

\author{
Ming Li $\cdot$ Kenneth W. Berendzen · Friedrich Schöffl
}

Received: 26 January 2010/ Accepted: 26 April 2010/Published online: 11 May 2010

(c) The Author(s) 2010. This article is published with open access at Springerlink.com

\begin{abstract}
The class A heat shock factors HsfA1a and HsfA1b are highly conserved, interacting regulators, responsible for the immediate-early transcription of a subset of heat shock genes in Arabidopsis. In order to determine functional cooperation between them, we used a reporter assay based on transient over-expression in Arabidopsis protoplasts. Reporter plasmids containing promoters of Hsf target genes fused with the GFP coding region were co-transformed with Hsf effector plasmids. The GFP reporter gene activity was quantified using flow cytometry. Three of the tested target gene promoters (Hsp25.3, Hsp18.1-CI, Hsp26.5) resulted in a strong reporter gene activity, with HsfA1a or HsfAlb alone, and significantly enhanced GFP fluorescence when both effectors were co-transformed. A second set of heat shock promoters (HsfA2, Hsp17.6CII, Hsp17.6C-CI) was activated to much lower levels. These data suggest that HsfA1a/1b cooperate synergistically at a number of target gene promoters. These targets are also regulated via the
\end{abstract}

Electronic supplementary material The online version of this article (doi:10.1007/s11103-010-9643-2) contains supplementary material, which is available to authorized users.

\section{Li · F. Schöffl $(\bowtie)$}

Zentrum für Molekularbiologie der Pflanzen (ZMBP),

Allgemeine Genetik, Universität Tübingen, Auf der

Morgenstelle 28, 72076 Tübingen, Germany

e-mail: friedrich.schoeffl@zmbp.uni-tuebingen.de

K. W. Berendzen

Zentrum für Molekularbiologie der Pflanzen (ZMBP),

Molekularbiologie der Pflanzen, Universität Tübingen, Auf der

Morgenstelle 5, 72076 Tübingen, Germany late HsfA2, which is the most strongly heat-induced class A-Hsf in Arabidopsis. HsfA2 has also the capacity to interact with HsfAla and HsfAlb as determined by bimolecular fluorescence complementation (BiFC) in Arabidopsis protoplasts and yeast-two-hybrid assay. However, there was no synergistic effect on Hsp18.1-CI promoter-GFP reporter gene expression when HsfA2 was co-expressed with either HsfA1a or HsfA1b. These data provide evidence that interaction between early and late HSF is possible, but only interaction between the early Hsfs results in a synergistic enhancement of expression of certain target genes. The interaction of HsfAla/A1b with the major-late HsfA2 may possibly support recruitment of HsfA2 and replacement of HsfA1a/A1b at the same target gene promoters.

Keywords Heat shock transcription factor .

Oligomerization domain - BiFC - Flow cytometry ·

Yeast-two-hybrid interaction $\cdot$ Protoplast transformation

\section{Introduction}

Heat shock factors (Hsfs) are central regulators of the heat shock response in eukaryotes. Hsfs are involved in stress sensing and signalling and can also be involved in the regulation of other cellular processes, including development. The functional properties of Hsfs are attributed to conserved structural domains: the highest degree of conservation is observed for the DNA-binding domain (DBD), which is composed of helix-turn-helix structures; and the oligomerization domain (OD), composed of hydrophobic heptad repeats. These structures are required for the formation of trimeric, high affinity DNA-binding complexes (Peteranderl and Nelson 1992; Peteranderl et al. 1999). 
Hsfs bind to the heat stress elements (HSE: 5'-nGAAnn TTCnnGAAn-3' or 5'nTTCnnGAAnnTTCn3') present in the upstream promoter regions of heat shock genes (for review see Schöffl et al. 1998).

Plants comprise a superfamily of Hsfs classified as class A-, -B, and -C Hsfs. Class B- and non-plant-Hsfs differ from class A- and C-Hsfs by an additional 21 or 7 amino acids, respectively, which separate the two subdomains HR-A and HR-B located in the hydrophobic regions (Nover et al. 1996, 2001). Furthermore, class B-Hsfs lack the acidic AHA motif, which has a crucial activator function in class A-Hsfs (Döring et al. 2000).

In Arabidopsis, 15 class A and 5 class B-Hsf genes have been identified. Members of class A have been characterized as transcriptional activators of heat shock genes. The Arabidopsis Hsfs AtHsfAla and AtHsfA1b are low-level constitutively expressed proteins, which become activated for DNA-binding and transcriptional simulation of heat shock target genes immediately upon heat stress (Lohmann et al. 2004; Busch et al. 2005). Mutational analysis showed that these two "early" Hsfs are functionally redundant (Lohmann et al. 2004) and the Hsf proteins interact with each other in vitro and in vivo (Li et al. 2010).

Interestingly, one of the so-called "late" class A Hsf genes, AtHsfA2, whose own expression is strongly enhanced upon heat stress, appears to regulate the expression of a set of target genes that largely overlaps with those regulated by AtHsfA1a and AtHsfA1b (Wunderlich et al. 2007). Thus the "early" constitutive Hsfs are considered as the primary regulators in the cell and may become replaced by late Hsfs which work thereafter throughout the duration of the heat stress response (Wunderlich et al. 2007). This is consistent with the finding that HsfA2 was essential for the duration, but not for the induction, of acquired thermotolerance (Charng et al. 2007).

In order to understand the regulation and function of early and late class A-Hsfs in Arabidopsis, we analysed their interaction using bimolecular fluorescence complementation (BiFC) in protoplasts with confocal microscopy and flow cytometry in addition to yeast-two-hybrid interaction assays. These analyses show for the first time that the early class A-Hsfs (HsfA1a and HsfA1b) can interact with the late HsfA2, and that the oligomerization domains are required and sufficient for this interaction. The combined activator functions of HsfAla and HsfA1b, when co-expressed as effectors in Arabidopsis protoplasts, lead to a synergistic enhancement of expression of a specific set of target promoter-reporter genes. Furthermore, co-expression of early and late Hsfs caused only additive effects when assaying for the induction of the Hsp18.1-CI promoter, which is a common target for both types of Hsfs.

\section{Materials and methods}

Protoplasts transformation, BiFC, and flow cytometric assays

Arabidopsis thaliana (Col-0) cell-culture growth, preparation and transformation of protoplasts were carried out as described by (Li et al. 2010).

According to Walter et al. (2004) Bimolecular Fluorescence Complementation (BiFC) was used to study the interaction between Hsf fusion-proteins. Each interaction partner to be tested is fused to $\mathrm{N}$ - or, respectively C-terminal parts of the Yellow Fluorescence Protein (YFP). After co-transformation and expression of the fusion proteins in Arabidopsis protoplasts, YFP-fluorescence is reconstituted if two proteins can stably interact with each other. The new BiFC constructs of the late HsfA2 (HsfA2$\mathrm{YFP}^{\mathrm{c}}$, HsfA2-YFP ${ }^{\mathrm{N} 152}$, and $\mathrm{OD}^{\mathrm{HsfA}}-\mathrm{YFP}^{\mathrm{N} 152}$ ) were constructed by cloning the full length HsfA2 protein coding region (PCR products from the start codon to the last C-terminal amino acid) or the oligomerization domain (OD; Supplement Tables 1, 4) into the XbaI and AscI sites of the BiFC vectors $p U C-S P Y C E$ and $p U C-S P Y N E-152$ as described by $\mathrm{Li}$ et al. (2010). The BiFC constructs of the early Hsfs (HsfA1a-YFP ${ }^{c}$, HsfA1b-YFP ${ }^{c}$ and the oligomerization domain deleted constructs HsfA1a $\Delta O D$ YFP $^{\mathrm{N} 152}$ and HsfA1a $\Delta$ OD-YFP ${ }^{\mathrm{C}}$ were previously described by Li et al. (2010).

Interactions between Hsf-YFP constructs were visualized 1 day after transfection by CLSM (Leica TCS SP2) by excitation at $488 \mathrm{~nm}$ and measuring fluorescence emitted at $594 \mathrm{~nm}$ according to $\mathrm{Li}$ et al. (2010). Fluorescence of protoplasts was quantified by flow cytometry using a MoFlo (Modular Flow; Beckman Coulter) as described by Li et al. (2010), using $488 \mathrm{~nm}$ excitation and emission captured in FL1 (505-554 nm) plotted against auto-fluorescence in FL2 (555-605 $\mathrm{nm})$. The fluorescence index was calculated based on the mean fluorescence multiplied by the fraction $(\%)$ of fluorescent cells. The results were calculated from three independent experiments.

Protein extraction, western blotting

The expression of Hsf-fusion proteins in transfected protoplasts was examined by Western blot analysis essentially as described by Li et al. (2010) using Myc-tag antibody (Cell Signaling) for detection of $\mathrm{YFP}^{\mathrm{N}}$ and HA antibody (Roche) for $\mathrm{YFP}^{\mathrm{C}}$.

Promoter GFP reporter assay

The promoter fragments of six genes [Hsp26.5-P(r), -18.1CI, -25.3-P, Hsp17.6C-CI, -17.6CII, HsfA2] included the 
5 'upstream regions (that is, starting immediately upstream of the start codon) were amplified by PCR using Pfu DNA Polymerase (Fermentas) and cloned into GFP reporter vector (pGKTan; Supplement Tables 3, 4). Three ug of each promoter construct was co-transformed with either $25 \mu \mathrm{g}$ of bZIP63 (non-Hsf-factor control) or the same amount of an Hsf-construct alone or in combination of two different Hsf-constructs; additionally, $5 \mu \mathrm{g}$ pBT835SLUCm3 vector was co-transformed with for normalization of the transformation efficiency (Sprenger-Haussels and Weisshaar 2000). Fifty $\mu \mathrm{g}$ of carrier DNA (sheared Herring sperm, Sigma) was added to all samples. The transfected protoplasts were heat-treated $\left(37^{\circ} \mathrm{C}, 3 \mathrm{~h}\right)$ followed by one hr recovery at room temperature $\left(25^{\circ} \mathrm{C}\right)$. Room temperature controls were incubated at $25^{\circ} \mathrm{C}$ during the entire time. The promoter-driven GFP fluorescence was quantified by flow cytometry as described above. From this we derived a specific GFP fluorescence index calculated as follows: growth on selective media according to the manufacturer's instructions (Clontech/Biosciences Matchmaker GAL4 Two-Hybrid System). Strength of interaction was examined by spotting serial dilutions $1: 10,1: 100$, and $1: 1000$ on the SD double dropout $(-\mathrm{L},-\mathrm{W})$ and the SD triple dropout (-L, -W, -H) plates. Yeast diploids containing each empty pGBKT7 or pGADT7 were used as negative controls.

\section{Results}

Nuclear localization and interaction between HsfA2, HsfA1a and HsfA1b

We employed BiFC to test whether the late-class A HSF HsfA2 can interact with the early HsfA1a and HsfA1b in vivo. Plasmids encoding YFP-fusion proteins with Hsfs were cloned into the $p U C-S P Y N E-152$ and $p U C-S P Y C E$, respectively, and were transformed into the Arabidopsis

GFP index $=\frac{\text { Hsf-dependent GFP fluorescence }(\text { RFU }) / \text { Luciferase activity }(\text { RLU } / \mu \mathrm{g} \text { protein } / \mathrm{s})}{\text { bZIP-dependent GFP fluorescence }(\mathrm{RFU}) / \text { Luciferase activity }(\mathrm{RLU} / \mu \mathrm{g} \text { protein/s })}$

Protein was isolated by vigorously vortexing protoplasts pellets in $100 \mu \mathrm{LUC}$ extraction buffer $(100 \mathrm{mM}$ potassium phosphate, $1 \mathrm{mM}$ DTT, pH7.5) for $30 \mathrm{~s}$. After centrifugation (10 $\mathrm{min}, 13,000 \mathrm{~g}, 4^{\circ} \mathrm{C}$ ), the supernatant was pipetted into a new $1.5 \mathrm{ml}$ Eppendorf tube and used for determination of LUC activity and protein concentrations. At room temperature, after adding $100 \mu$ filter-sterilized LUC assay buffer (20 mM tricine, $1.07 \mathrm{mM}\left(\mathrm{MgCO}_{3}\right)_{4} \mathrm{Mg}(\mathrm{OH})_{2} \times 5 \mathrm{H}_{2} \mathrm{O}, 2.67$ $\mathrm{mM} \mathrm{MgSO} 4,0.1 \mathrm{mM}$ EDTA, $33 \mathrm{mM}$ DTT, $0.27 \mathrm{mM}$ CoA, $0.47 \mathrm{mM}$ D-luciferin, $0.53 \mathrm{mM}$ ATP, $\mathrm{pH} 7.8$ ) to $10 \mu \mathrm{l}$ of extract LUC activity was determined by Luminometer Lumat LB9501 with an interval of $10 \mathrm{~s}$. Specific LUC activity is given as relative light units (RLUs) $\mu$ g protein-1 s-1.

\section{Yeast-two-hybrid interaction assay}

Full length coding sequences of Arabidopsis HsfA2, HsfA1a, and HsfA1b were amplified by PCR using Pfu DNA Polymerase (Fermentas) and cloned into pGBKT7 bait vector (fused with Gal4 DNA binding domain) and pGADT7 prey vector (fused with GAL4 activation domain (Supplement Tables 2, 4). The constructs were verified by custom sequencing (AGOWA), and transformed into yeast Y187 and AH109. Homologous or heterologous Hsf interactions were identified by testing combinations of Hsfbait and Hsf-prey constructs co-expressed in yeast. Interactions between Hsf fusion proteins were detected by protoplasts ( $\mathrm{Li}$ et al., 2010). BiFC indicating homomeric interaction of HsfA2-YFP ${ }^{\mathrm{N} 152}$ with HsfA2-YFP ${ }^{\mathrm{C}}$ was clearly observed in the nucleus and less in the cytoplasm (not shown). The heterologous combination of HsfA2YFP $^{\mathrm{N} 152}$ with HsfA1a-YFP ${ }^{\mathrm{C}}$ or HsfA1b-YFP ${ }^{\mathrm{C}}$ also exhibited BiFC in the nucleus (not shown). Flow cytometric quantification of BiFC signals (Fig. 1d) showed a relatively strong fluorescence for homomeric (HsfA2, pair \#1) and somewhat weaker fluorescence for heteromeric interactions of HsfA2 with HsfA1a (pair \#2) or HsfA1b (pair \#3; Fig. 1a).

In order to examine whether the ODs of the interacting Hsfs are required and sufficient for the interactions, BiFC vectors expressing only the ODs of HsfAla, HsfA1b (Li et al. 2010) or HsfA2 fused with YFP constructs were tested (Fig. 1c). The heteromeric interaction between HsfA2 and was not detectable when the HsfA1a $\Delta O D-Y_{F P}{ }^{C}$ construct lacking the OD was used in any combination with HsfA2 (Fig. 1a, d, pairs \#4 and \#5). Heteromeric OD combinations of HsfA2 and HsfA1a (pair \#2: OD ${ }^{\text {HsfA1a }}$ $\mathrm{YFP}^{\mathrm{N} 152} / \mathrm{OD}^{\mathrm{HsfA}}-\mathrm{YFP}^{\mathrm{C}}$ ) or respectively HsfA1b (pair \#3: $\mathrm{OD}^{\mathrm{HsfA} 1 \mathrm{~b}}-\mathrm{YFP}^{\mathrm{N} 152} / \mathrm{OD}^{\mathrm{HsfA}}-\mathrm{YFP}^{\mathrm{C}}$ ) interacted with each other to about the same level as the full length Hsfs (Fig. 1b). The homomeric interaction between the ODs of HsfA2 (pair 1: OD ${ }^{\mathrm{HsfA} 2}-\mathrm{YFP}^{\mathrm{N} 152} / \mathrm{OD}^{\mathrm{HsfA} 2}-\mathrm{YFP}^{\mathrm{C}}$ ) appeared to be significantly less effective as compared with the full length HSF-constructs (Fig. 1b), suggesting that either the 

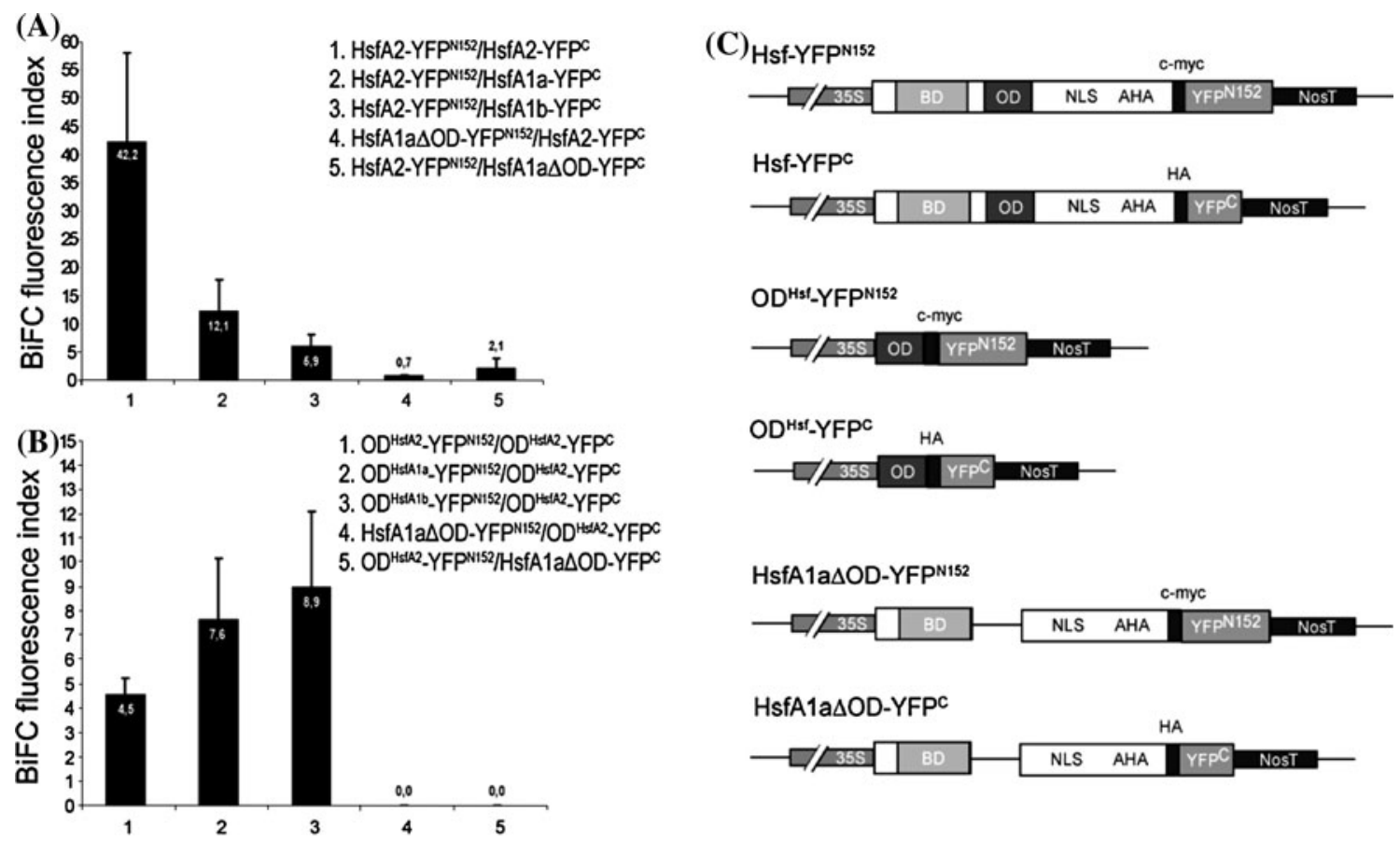

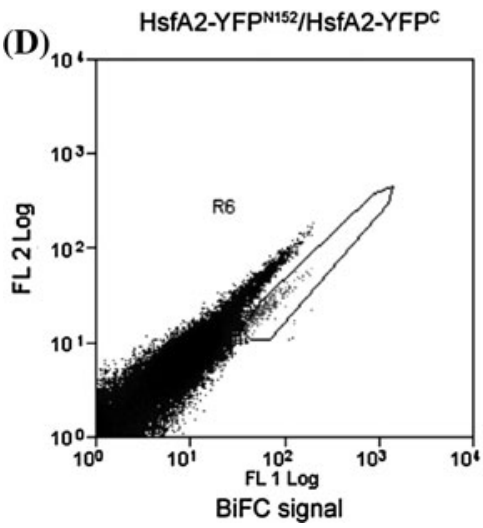

Fig. 1 Quantification of interactions between Late HsfA2 and early HsfA1a and HsfA1b. a BiFC Interactions between full-length HsfYFP constructs in Arabidopsis protoplasts. No interaction was detected between full length HsfA2 and HsfA1a- $\triangle O D$ fusion protein. b Interactions between the oligomerization domains $(O D)$ of HsfA2, HsfA1a and HsfA1b YFP fusion proteins in protoplasts. No interaction was detected between any OD-YFP construct and HsfA1a- $\Delta O D-$ YFP constructs. The BiFC was quantified by flow cytometric analysis (see "Methods"). c Schematic diagrams of types of Hsf-YFP constructs, drawings are not to scale. $B D$ DNA binding domain,

entire OD domain was not taken, or additional amino acids participate in homomeric HsfA2 interaction. This is further supported by the data presented in Fig. 1a, where a deletion of the OD domain did not completely abolish binding. Conversely, no fluorescence was obtained for heteromeric interactions between OD and the OD-deleted HsfA1a fusion proteins $\left(H s f A 1 \mathrm{a} \Delta \mathrm{OD}-\mathrm{YFP}^{\mathrm{C}}\right.$ : pairs \#4 and \#5). As observed previously for HsfAla/Alb interaction, the homologous and heterologous interactions with HsfA2 took already place in the absence of heat stress and there was no significant change in the sub-cellular localization and intensity of BiFC (data not shown).

Finally, we had used yeast-two-hybrid assays for re-examining and confirming the interactions between HsfA2, HsfA1a, and HsfA1b. Binding domain (BD) and activation domain (AD) versions of GAL4-Hsf constructs 


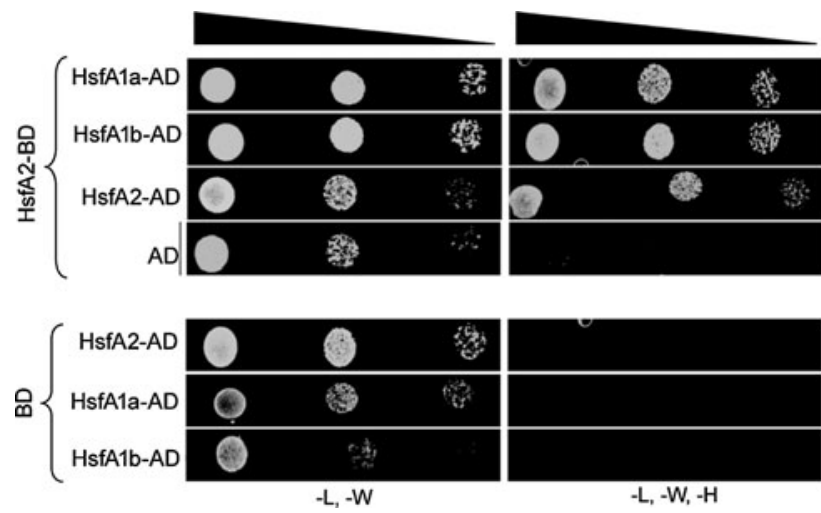

Fig. 2 Yeast-two-hybrid interactions between early (HsfA2) and late (HsfA1a and HsfA1b) class A-Hsfs. The interaction between GAL4 bait ( $B D$ binding domain) and prey ( $A D$ activation domain) HSF fusion proteins in yeast was assayed by spotting serial dilutions (1:10, 1:100, 1:1000) of yeast on selective dropout media: -L (leucine), -W (tryptophan) $-\mathrm{H}$ (histidine)

were generated (Supplement Table 2) and assayed for interaction in the combinations depicted in Fig. 2. The homologous combination (HsfA2-BD/HsfA2-AD) and both heterologous combinations (HsfA2-BD/HsfA1a-AD and HsfA2-BD/HsfA1b-AD) resulted in the same growth patterns on selective media thus indicating interaction. Using the overlay assay, significant levels of $ß$-gal activity were detected (data not shown).

Promoter specificity of HsfA1a/A1b and expression of target genes

In order to assess whether interaction of HsfAla and HsfA1b may influence the promoter specificity and activity of target genes, two groups of target gene promoters were chosen for the analysis: HsfA1a/A1b-dependent [Hsp26.5$P(r), \quad-18.1-C I, \quad-25.3-P]$ and HsfA1a/1b-independent [Hsp17.6C-CI, -17.6CII, HsfA2] promoters. The discrimination of these promoters is based on the expression profiling data of Hsf knock out mutants (Busch et al. 2005). All the tested genes, with the exception of $H s f A 2$, are strongly heat-induced Heat Shock Protein (Hsp) genes of Arabidopsis. The promoters (p) of these genes were cloned into GFP reporter vector pGTKan. The reporter plasmids were transformed into Arabidopsis protoplasts along with effector plasmids encoding different Hsfs-YFP ${ }^{\mathrm{N} 152}$ constructs driven by $C a M V-35 S$ promoter (same as used for BiFC analyses). The dummy effector construct 35S-bZIP63 was used as a control for normalizing Hsf effector-dependent reporter gene activation. Luciferase expression plasmid (pBT8-35SLUCm3), co-transformed with the reporter/effector plasmids, was used for normalizing transfection efficiency according to SprengerHaussels and Weisshaar (2000). The reporter and effector constructs are listed in Supplement Tables 1 and 3. All reporter constructs were tested in the presence and absence of heat shock stress with the four effectors: bZIP63 as negative control, HsfA1a alone, HsfA1b alone, or combined HsfA1a/HsfA1b.

Each transfected protoplast sample was split, one aliquot was treated by heat stress $\left(37^{\circ} \mathrm{C}, 3 \mathrm{~h}\right)$ and the other was kept at the control temperature $\left(25^{\circ} \mathrm{C}, 3 \mathrm{~h}\right)$. The GFP fluorescence was recorded for each sample using flow cytometry and a GFP-index was calculated to derive the promoter induction. In the absence of specific pHspeffectors (i.e. bZIP63) the GFP fluorescence index of all reporter constructs increased upon heat stress by factors of

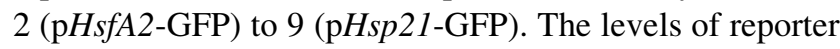
activities obtained with the dummy effector bZIP63 still responded to heat stress. This increase in the dummy effector bZIP63 controls is attributed to the heat activation of the endogenous Hsfs, which are present in all cells and also capable to recognize and activate transcription of the heat shock promoter-reporter gene construct.

Without heat stress, HsfA1a and HsfA1b caused higher levels of reporter activity of all pHsp-driven GFP-constructs with the exception of pHsfA2-GFP. Upon heat stress, the levels of HsfA1a/1b dependent reporter activities increased in the presence of either HsfA1a or HsfA1b alone

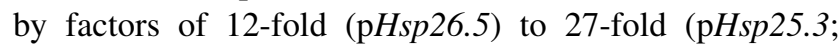
Fig. 3). In contrast, HsfA1a or HsfA1b caused only minor increases of reporter activities of HsfAla/1b-independent

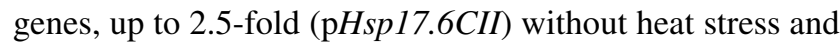

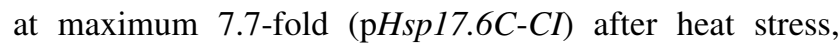
respectively.

The combined co-expression of HsfAla and HsfA1b, however, resulted in somewhat higher levels of reporter activity for all tested promoter-reporter genes compared to HsfA1a or HsfA1b alone, again except for pHsfA2-GFP. Comparison of the basal (RT) and the heat shock induced expression of $\mathrm{pHsps} / \mathrm{HsfA2}-\mathrm{GFP}$ activities indicate significantly higher levels of pHsp-GFP activities. Based on the background control, bZIP63 transformation at normal temperature, the GFP activity of the three HsfA1a/1bdependent genes increased 18.8-fold (pHsp26.5), 30.3-fold (pHsp18.1-CI) and 38.4-fold (pHsp25.3), respectively. The highest level of GFP activity of the HsfA1a/1b-independent genes increased to only about 10.9-fold (pHsp17.6C-CI). These differences in reporter gene activities, particularly after heat stress, are in good agreement with the previous classification of HsfA1a/A1b-dependent and independent genes (Busch et al. 2005). Moreover, cooperation between the two Hsf effectors HsfA1a and HsfA1b on the expression of HsfA1a/A1b-dependent genes [Hsp26.5-P(r), -18.1-CI, $-25.3-P]$ indicates that the heteromeric interaction causes a synergistic effect; the values of reporter activities are significantly higher $(P \leq 0.05)$ compared to single HsfA1a- or 


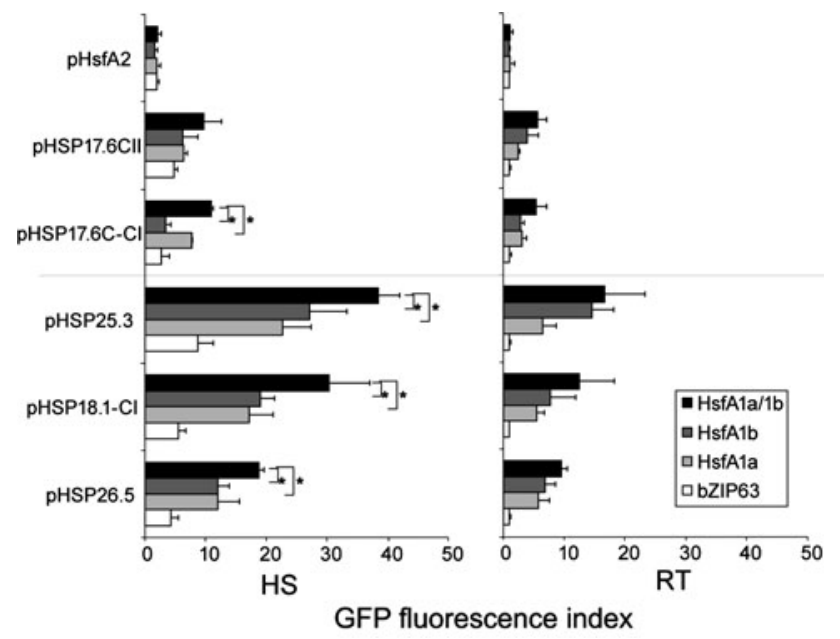

Fig. 3 Promoter specificity of HsfAla/A1b and expression of target genes. Homomeric (HsfA1a, HsfA1b) or heteromeric (HsfA1a/1b) effectors coexpressed in Arabidopsis protoplasts together with HsfA1a/1b-independent [top panels: $H s p 17.6 C-C I$, -17.6CII, HsfA2] and HsfA1a/1b-dependent [bottom panels: $H s p 26.5-P(r),-18.1-C I$, $-25.3-P]$ promoter-driven GFP reporter genes. GFP fluorescence was quantified by flow cytometry. Three ug of each promoter::reporter construct were cotransformed with $25 \mu \mathrm{g}$ effector DNA (for double HSF transformations $12.5 \mu \mathrm{g}$ of each construct). Data were normalized for transformation efficiency using co-transformation of $5 \mu \mathrm{g}$ luciferase $(L U C)$ expression plasmid. The bZIP effector expression was used as a negative control for determining endogenous Hsfbackground activities in protoplasts. The expression of the effector proteins was determined by Western Blots (see supplement Fig. 1). HS (left half panels): heat stress treatment $\left(3 \mathrm{~h}, 37^{\circ} \mathrm{C}\right)$; RT (right half panels): room temperature $\left(3 \mathrm{~h} 25^{\circ} \mathrm{C}\right)$. Significance indicated by an asterisk, $P \leq 0.05$. (Hsp17.6C-CI: HsfA1a vs. HsfA1a/1b $t$-test $P=0.002$, HsfA1b vs. HsfA1a/1b $t$-test $P=0.001$; Hsp25.3-P: HsfAla vs. HsfAla/lb $t$-test $P=0.009$, HsfAlb vs. HsfA1a/1b $t$-test $P=0.05$; Hsp18.1-CI: HsfA1a vs. HsfA1a/1b $t$-test $P=0.04$, HsfA1b vs. HsfA1a/1b $t$-test $P=0.02$; Hsp26.5: HsfA1a vs. HsfA1a/1b $t$-test $P=0.03$, HsfAlb vs. HsfA1a/1b $t$-test $P=0.002$ )

HsfAlb-driven reporter gene activities. Reporter gene activity

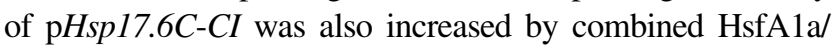
$\mathrm{A} 1 \mathrm{~b}$ effector expression, unlike the other two of HsfAla/A1b-

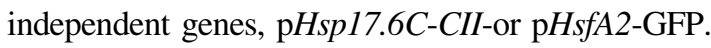

The extremely low levels of reporter activities of $\mathrm{pH} f$ A2-GFP construct are not so surprising. Expression profiling data showed also a much weaker heat stress induction of HsfA2 expression which is $\sim 20$-fold lower than the expression of the strongly induced sHsp-genes (Busch et al. 2005). Thus the HsfA2 promoter appears to be "weaker" compared to other HsfAla/A1b-independent heat shock genes. At present it is unknown which Hsf is the natural transcriptional activator of Arabidopsis HsfA2.

Early and late Hsfs can act on the regulation of $\mathrm{pHsp18.1-CI-GFP}$ reporter activity

The interaction between early (HsfAla/A1b) and late (HsfA2) Hsfs suggests that they might also functionally

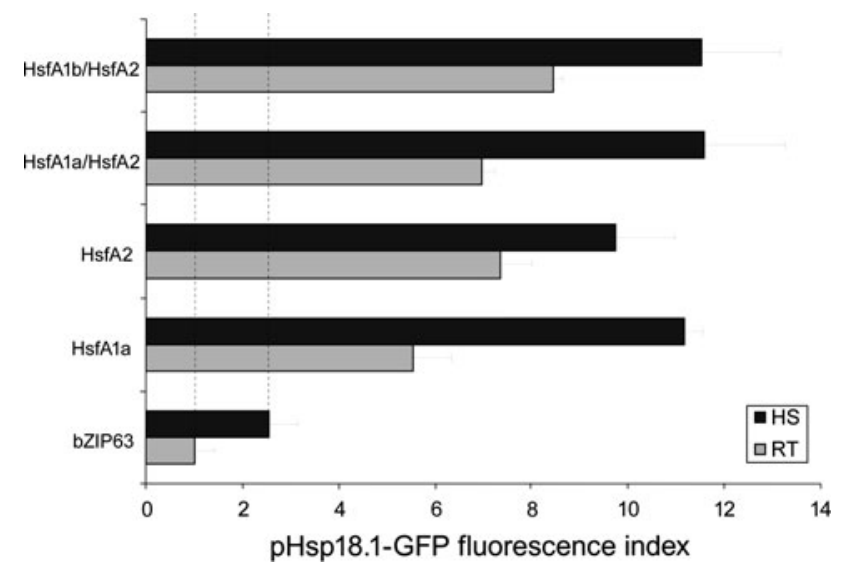

Fig. 4 Hsp18.1 promoter activation by early and late class A Hsfs. Homomeric (HsfA1a, HsfA2) or heteromeric (HsfA1a/HsfA2, HsfA1b/HsfA2) effectors coexpressed in Arabidopsis protoplasts together the Hsp18.1-CI promoter-driven GFP reporter gene. GFP fluorescence was quantified by flow cytometry. The expression of the effector proteins was determined by Western Blots (see supplement Fig. 1). Three ug of the promoter-reporter construct were cotransformed with $25 \mu \mathrm{g}$ effector DNA (for double HSF transformations $12.5 \mu \mathrm{g}$ of each construct). Data were normalized for transformation efficiency using co-transformation of $5 \mu \mathrm{g}$ luciferase (LUC) expression plasmid. The bZIP effector expression was used as a negative control for determining endogenous HSF-activities of protoplasts (see supplement Fig. 3). $H S$ heat stress treatment $\left(3 \mathrm{~h}, 37^{\circ} \mathrm{C}\right), R T$ room temperature $\left(3 \mathrm{~h} 25^{\circ} \mathrm{C}\right)$

cooperate in promoter activation of their common target genes. In order to analyze such an effect we tested the common target pHsp18.1-CI-GFP-reporter construct. Following co-transformation of protoplasts with $\mathrm{pHsp18.1-CI-}$ GFP-reporter and effector plasmids (bZIP63, HsfA1a alone, HsfA2 alone, HsfA1a/A2 or HsfA1b/A2 combinations), significant GFP activity was measured compared to the dummy bZIP63 effector (Fig. 4). In all cases, heat shock resulted in enhanced promoter activation, but there were no significant differences due to the simultaneous presence of early and late Hsfs. This demonstrated that early and late Hsfs have the same potential to recognize the same heat shock promoters and regulate the expression of those genes, yet in this experimental setup, there was no indication for synergistic cooperation between them.

\section{Discussion}

A partial overlap between sets of putative target genes regulated by AtHsfA1a/1b early and AtHsfA2 late in the heat stress response was indicated by the transcriptome analysis of HSF knock out mutants (Nishizawa et al. 2006; 2009; Schramm et al. 2006; Charng et al. 2007). We have previously demonstrated that the early HsfAla and HsfAlb can interact with each other ( $\mathrm{Li}$ et al. 2010). In the present paper, we show for the first time in Arabidopsis that these 
two early-Hsfs can also interact with the late-HsfA2 in vivo. The interaction occurred predominantly in the nucleus, but the interaction was not dependent on heat stress. We would like to point out that in these interaction studies performed in Arabidopsis protoplasts the original split $\mathrm{YFP}^{\mathrm{N}}$ vector that has some non-specific complementation activity could not be used without N-terminal modifications $\left(\mathrm{YFP}^{\mathrm{N} 152}\right.$ : Li et al. 2010), while the original $\mathrm{YFP}^{\mathrm{N}}$ construct had been used for studying Hsf interactions in the tobacco system (Baniwal et al. 2007). This and other discrepancies indicate that tobacco and Arabidopsis protoplast systems seem to have different properties.

The interactions between HsfAla and HsfA1b (Li et al. 2010) and they with HsfA2 (this paper) require their ODs. The OD-deleted version of HsfAla was unable to show interaction with any full length Hsf or OD constructs. It cannot be excluded that other parts of Hsfs may also be involved in protein-protein interaction, but from these results, we conclude not to any major extent. An OD-dependent interaction suggests that hetero-oligomeric complexes may be formed. An interaction, that depends on the oligomerization domains, is reminiscent of the interaction between tomato LpHsfA1 and LpHsfA2 (ChanSchaminet et al. 2009) and between AtHsfA4 and AtHsfA5 (Baniwal et al. 2006). In both cases the interactions are highly specific for the respective Hsf pair. The early LpHsfA1, described as a master regulator of the HS response in tomato, triggers the expression of LpHsfA2 and LpHsf B1 that seem to cooperate in modulating gene expression to maintain thermotolerance. The highly specific interactions between AtHsfA4 and AtHsfA5 have an adverse negative effect on the transcription activation activity of AtHsfA4.

The situation in Arabidopsis appears to be different; no Hsf has been identified as a master regulator of the heat shock response. The early Hsfs AtHsfAla and AtHsfA1b cooperate upon heat stress resulting in a strong and rapid expression of only subgroups of Hsps and Hsfs. The expression of the late AtHsfA2 is not controlled by HsfA1a/A1b, since its heat-induced expression is largely unaffected in $h s f A l a / A l b$ knock out plants (Busch et al. 2005). As such, the mode of activation of HsfA2 expression in Arabidopsis is as yet unknown. Consistent with what is described in the literature, the late Hsfs contribute only little to the initial early expression of heat shock genes. Later in the heat shock response of Arabidopsis, a transition from early to late phase takes place. This stage is marked by a transition in the pattern of Hsf-DNA-binding capacity (Lohmann et al. 2004) and the massive heatinduced expression of Hsps and other Hsfs. The formation of the major-late HSF-DNA binding complex that replaces the early HsfA1a/1b-dependent complex, requires HsfA2 and suggests that HsfA2 is dominating the late phase of the HS response (Wunderlich et al. 2007).
During the early-late phase transition of the heat shock response there must be timely and spatial overlap between early and late Hsf functions. Since we find interaction between early and late Hsfs but no cooperation and enhancement of target gene expression, the interaction may reflect the recruitment of HsfA2 to the same promoters previously activated by HsfA1a/A1b. Indeed, HsfA2 has been shown to be required for sustaining heat-induced gene expression but not initiating it (Nishizawa-Yokoi et al. 2009; Charng et al. 2007). AtHsfA2 appears to be a rather stable protein, which once accumulated, has an enduring effect as a transcriptional activator. The rapid decline of its putative target gene transcripts in the recovery phase (Schramm et al. 2006) indicates that HsfA2 becomes rapidly inactivated during recovery. At this stage HsfA2 might be retained in the cytoplasm in complexes with heat shock proteins such as it has been shown for tomato LpHsfA2 (Port et al. 2004; Heerklotz et al. 2001); correspondingly, we have observed interaction of HsfA2 with HsfA1a/A1b also in the cytoplasm.

These examples show that some of the basic principles regulating Hsf activities and target gene expression are conserved in different plant species. However, convergent evolution of Hsf genes has taken place as indicated by functional differences (molecular interactions and target gene expression) of the strongly sequence-conserved Hsfs LpHsfB1 (Bharti et al. 2004) and AtHsfB1 (Kumar et al. 2009) or LpHsfA2 (Chan-Schaminet et al. 2009) and AtHsfA2 (this paper).

Our promoter transactivation experiments using only GFP as the reporter are largely consistent with previously published literature for the HsfA1a/A1b-dependent promoters, which suggested synergistic activation by these two early Hsfs (Lohmann et al. 2004, Busch et al. 2005). Although GFP has been used before as a promoter expression reporter (Ducrest et al. 2002; Bargmann and Birnbaum 2009); no previous study standardized a protocol for multiple transactivation experiments for quantitative comparisons. In this study, we were not able to measure strong heat induced expression of all of the HsfA1a/A1bdependent promoters, which was expected when observing their expression data (Busch et al. 2005; Wunderlich et al. 2007). These promoters appear heat-inducible but to a much lower extent than previously published. The relatively lower levels of heat induction are a consequence of the already very high levels of reporter gene activities at normal temperature. The high constitutive levels at normal temperature in Arabidopsis protoplasts reflect a de-repression of Hsf-activities upon transient overexpression leading to multimeric, and probably trimeric complexes as previously shown for HsfAla when over-expressed in $E$. coli (Hübel and Schöffl 1994) or Drosophila (Hübel et al. 1995). This phenomenon is attributed to the titration of 
negatively interacting factors (Shi et al. 1998) and is consistent with the results of stably transformed Hsfoverexpressing transgenic plants (Lee et al. 1995; Prändl et al. 1998).

Nevertheless, in this protoplast assay, the HsfA1a/A1bdependent promoters $[H s p 26.5-P(r),-18.1-C I,-25.3-P]$ resulted in much stronger reporter gene activities than HsfA1a/1b-independent [Hsp17.6C-CI, -17.6CII, HsfA2] promoters. These findings are consistent with a promoter specificity and synergistic interaction of HsfAla and HsfA1b for only a subset of the heat shock gene promoters. The HsfA1a/A1b-independent promoters are also recognized and activated by HsfAla and HsfAlb in the protoplast assay but to lower levels and not in a synergistic fashion. Similar to in vitro experiments, expression in protoplasts leads to high levels of active (de-repressed) Hsfs that may always recognize and bind to the HSE-elements present in the promoter regions of heat shock genes.

In contrast to early LpHsfA1 and late LpHsfA2 interaction, there was no synergistic activation of target gene promoters by AtHsfA1a/1b and HsfA2. A moderated synergistic effect was only observed on a subset of heat shock target gene-reporter constructs when the early HsfAla and HsfA1b were co-expressed. The synergistic effects were much lower (1-2-fold) compared to the relatively strong synergism described for the interaction between tomato LpHsfA1 and LpHsfA2 (Chan-Schaminet et al. 2009). However, as discussed above, the Arabidopsis HsfAla/ HsfA1b are not true homologues LpHsfA1.

In conclusion: the Arabidopsis protoplast assay system provides a general and quick tool for studying the effects of Hsf interaction and trans-activation of heat shock gene expression. However, as evident for all systems working with transient overexpression of regulatory proteins (transcription factors), it cannot always truly reflect the in vivo situation. The wasteful overexpression of the effectors (Hsfs) and the undetermined state of chromatin structure of the target promoter-reporter plasmids in the cell may interfere with the natural processes regulating the activity of Hsf and gene expression. Thus conclusions from such experiments are only relevant if substantiated by data from other independent experimental approaches. In our present study the transient promoter-effector assays confirmed the Hsf-promoter specificities of heat shock genes, which were previously suggested by the analyses of Hsf gene knock out mutants and expression profiling data.

Acknowledgments We thank Caterina Brancato (ZMBP-Transformation Unit) for the preparation of protoplasts and excellent support, Drs. K. Harter and K. Schumacher (ZMBP-Pflanzenphysiologie) for vectors bZIP63-YFP ${ }^{\mathrm{N} 152}$, pGTKan, and pHsp18.1-CI-GFP. The research was funded by grants of the Deutsche Forschungsgemeinschaft (SFB446, project A2).
Open Access This article is distributed under the terms of the Creative Commons Attribution Noncommercial License which permits any noncommercial use, distribution, and reproduction in any medium, provided the original author(s) and source are credited.

\section{References}

Baniwal SK, Chan KY, Scharf K-D, Nover L (2006) Role of heat stress transcription factor HsfA5 as specific repressor of HsfA4. J Biol Chem. 282:3605-3613

Bargmann BO, Birnbaum KD (2009) Positive fluorescent selection permits precise, rapid, and in-depth overexpression analysis in plant protoplasts. Plant Physiol 149:1231-1239

Bharti K, von Koskull-Döring P, Bharti S, Kumar P, TintschlKorbitzer A, Treuter E, Nover L (2004) Tomato heat stress transcrition factor HsfB1 represents a novel type of general transcription coactivator with a histone-like motif interacting with the plant CREB binding protein ortholog HAC1. Plant Cell 16:1521-1535

Busch W, Wunderlich M, Schöffl F (2005) Identification of novel heat shock factor dependent genes and biochemical pathways in Arabidopsis thaliana. Plant J 41:1-14

Chan-Schaminet KY, Baniwal SK, Bublak D, Nover L, Scharf K-D (2009) Specific interaction between tomato HsfA1 and HsfA2 creates hetero-oligomeric superactivator complexes for synergistic activation of heat stress gene expression. J Biol Chem 284:20848-20857

Charng YY, Liu HC, Liu NY, Chi WT, Wang CN, Chang SH, Wang TT (2007) A heat-inducible transcription factor, HsfA2, is required for extension of acquired thermotolerance in Arabidopsis. Plant Physiol 143:251-262

Döring P, Treuter E, Kistner C, Lyck R, Chen A, Nover L (2000) The role of AHA motifs in the activator function of tomato heat stress transcription factors HsfA1 and HsfA2. Plant Cell 12:265-278

Ducrest AL, Amacker M, Lingner J, Nabholz M (2002) Detection of promoter activity by flow cytometric analysis of GFP reporter expression. Nucleic Acids Res 30(14):e65

Heerklotz D, Döring P, Bonzelius F, Winkelhaus S, Nover L (2001) The balance of nuclear import and export determines the intracellular distribution of tomato heat stress transcription factor HsfA2. Molecular Cell Biology 21:1759-1768

Hübel A, Schöffl F (1994) Arabidopsis heat shock factor: isolation and characterization of the gene and the recombinant protein. Plant Mol Biol 26:353-362

Hübel A, Lee JH, Wu C, Schöffl F (1995) Arabidopsis heat shock factor is constitutively active in animal and human cells. Mol Gen Genet 348:136-141

Kumar M, Busch W, Birke H, Kemmerling B, Nürnberger T, Schöffl F (2009) Heat shock factors HsfB1 and HsfB2b are involved in the regulation of Pdf1.2 expression and pathogen resistance in Arabidopsis. Molecular Plant 2:152-165

Lee JH, Hübel A, Schöffl F (1995) Derepression of the activity of genetically engineered heat shock transcription factor causes constitutive synthesis of heat shock proteins and increased thermotolerance in transgenic Arabidopsis. Plant J 7:603-612

Li M, Doll J, Weckermann K, Oecking C, Berendzen K-W, Schöffl' F (2010) Detection of in vivo interactions between Arabidopsis class A-HSFs, using a novel BiFC fragment, and identification of novel class B-HSF interacting proteins. Eur J Cell Biol 89:126132. doi:10.1016/j.ejcb.2009.10.012

Lohmann C, Eggers-Schumacher G, Wunderlich M, Schöffl F (2004) Two different heat shock transcription factors regulate 
immediate early expression of stress genes in Arabidopsis. Mol Gen Genom 271:11-21

Nishizawa A, Yabuta Y, Yoshida E, Maruta T, Yoshimura K, Shigeoka S (2006) Arabidopsis heat shock transcription factor A2 as a key regulator in response to several types of environmental stress. Plant J 48:535-547

Nishizawa-Yokoi A, Yoshida E, Yabuta Y, Shigeoka S (2009) Analysis of the regulation of target genes by an Arabidopsis heat shock transcription factor HsfA2. Biosci Biotechnol Biochem 73:890-895

Nover L, Scharf K-D, Gagliardi D, Vergne P, Czarnecka-Verner E, Gurley WB (1996) The Hsf world: classification and properties of plant heat stress transcription factors. Cell Stress Chaperones $1: 215-223$

Nover L, Bharti K, Döring P, Mishra SK, Ganguli A, Scharf K-D (2001) Arabidopsis and the heat stress transcription factor world: how many heat stress transcription factors do we need? Cell Stress Chaperones 6:177-189

Peteranderl R, Nelson HCM (1992) Trimerization of the heat stress transcription factor by a triple-stranded alpha-helical coiled-coil. Biochem 31:12272-12276

Peteranderl R, Rabenstein M, Shin Y, Liu CW, Wemmer DE, King DS, Nelson HCM (1999) Biochemical and biophysical characterization of the trimerization domain from the heat stress transcription factor. Biochem 38:383559-383569

Port M, Tripp J, Zielinski D, Weber C, Heerklotz D, Winkelhaus S, Bublak D, Scharf K-D (2004) Role of Hsp17.4-CII as coregulator and cytoplasmic retention factor of tomato heat stress transcription factor HsfA2. Plant Physiol 135:1457-1470
Prändl R, Hinderhofer K, Eggers-Schumacher G, Schöffl F (1998) HSF3, a new heat shock factor from Arabidopsis thaliana, derepresses the heat shock response and confers thermotolerance when overexpressed in transgenic plants. Mol Gen Genet 258:269-278

Schöffl F, Prändl R, Reindl A (1998) Regulation of the heat-shock response. Plant Physiol 117:1135-1141

Schramm F, Ganguli A, Kiehlmann E, Englich G, Walch D, von Koskull-Döring P (2006) The heat stress transcription factor HsfA2 serves as a regulatory amplifier of a subset of genes in the heat stress response in Arabidopsis. Plant Mol Biol 60: $759-772$

Shi S, Mosser DD, Morimoto RE (1998) Molecular chaperones as Hsf1-specific transcriptional repressors. Genes Dev 12:654-666

Sprenger-Haussels M, Weisshaar B (2000) Transactivation properties of parsley proline-rich bZIP transcription factors. Plant J 22:1-8

Walter M, Chaban C, Schutze K, Batistic O, Weckermann K, Nake C, Blazevic D, Grefen C, Schumacher K, Oecking C, Harter K, Kudla J (2004) Visualization of protein interactions in living plant cells using bimolecular fluorescence complementation. Plant J 40:428-438

Wunderlich M, Doll J, Busch W, Kleindt CK, Lohmann C, Schöffl F (2007) Heat shock factors: regulators of early and late functions in plant stress response. Plant Stress 1:16-22 